Archive for

Organic Chemistry

Arkivoc 2019, part vi, 158-166

\title{
A three-component procedure for the synthesis of 5-(1-aryl-3-arylprop-2-yn-1-yl)-2,2-butylidene-1,3-dioxane-4,6-dione derivatives
}

\author{
Zhaohui $\mathrm{Xu}^{\mathrm{a} *}$, Chenliang Zhou ${ }^{\mathrm{b}}$, Zhengbing $\mathrm{Xiang}^{\mathrm{c}}$, and Qiang Xiao ${ }^{\mathrm{c} *}$ \\ ${ }^{a}$ Department of Chemistry and Chemical Engineering, Jiangxi Normal University, Nanchang 330022, China \\ ${ }^{b}$ School of Foreign languages and Literature, Wuhan University, Wuhan 430072, China \\ ${ }^{c}$ Department of Orthopaedics, Jiangxi Provincial People's Hospital, Nanchang 330006, China \\ Email:002776@jxnu.edu.cn; doctorxiaoq@163.com
}

Received 08-19-2019

Accepted 09-29-2019

Published on line 10-26-2019

\section{Abstract}

A simple and efficient procedure for the synthesis of 5-(1-aryl-3-arylprop-2-yn-1-yl)-2,2-butylidene-1,3dioxane-4,6-dione derivatives through one-pot reactions of araldehydes, 2,2-butylidene-1,3-dioxane-4,6-dione and an arylethyne in the presence of $\mathrm{Cu}(\mathrm{OAc})_{2} \cdot \mathrm{H}_{2} \mathrm{O} / \mathrm{Na}$-D-isoascorbate, is described. The procedure involves initial Knoevenagel reaction, followed by conjugate addition. The high isolated yields, broad substrate scope, mild conditions, and easy operation are the main advantages of the protocol.
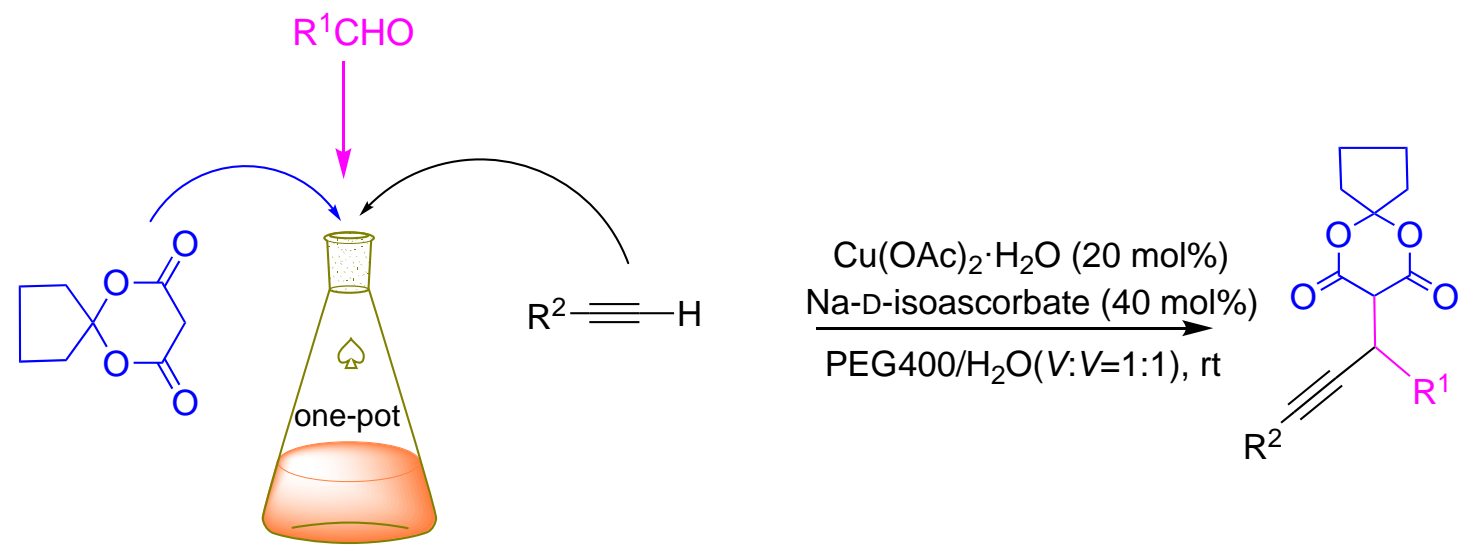

Keywords: B-alkynyl Meldrum's acid analogues, one-pot reaction, $\mathrm{Cu}(\mathrm{OAc})_{2} \cdot \mathrm{H}_{2} \mathrm{O} / \mathrm{Na}$-D-isoascorbate, 2,2butylidene-1,3-dioxane-4,6-dione 


\section{Introduction}

B-Alkynyl Meldrum's acid analogues have exhibited an amazingly wide spectrum of biological properties including as PDE IV inhibitors, TNF inhibitors, GPR40 receptor agonists, and GRP receptor antagonists. ${ }^{1,2}$ They are also important building blocks in organic synthesis performed to access diverse 6 -alkynyl carbonyl compounds, ${ }^{3} \gamma$-butyrolactones ${ }^{4-6}$ and clausenamide alkaloids. ${ }^{7}$ Therefore, the development of a simple and efficient methology for the synthesis of 6 -alkynyl Meldrum's acids has attracted the attention of synthetic as well as medicinal chemists.

5-(1-aryl-3-arylprop-2-ynyl)-2,2-methyl-1,3-dioxane-4,6-diones are commonly synthesized employing one of three methods involving conjugate addition of metalated teminal alkynes, in situ generated copper alkynylides or in situ generated zinc alkynylides to Meldrum's acid derived acceptors (Scheme 1).

(a) The conjugate addition of metalated teminal alkynes with Meldrum' s acid derived acceptors

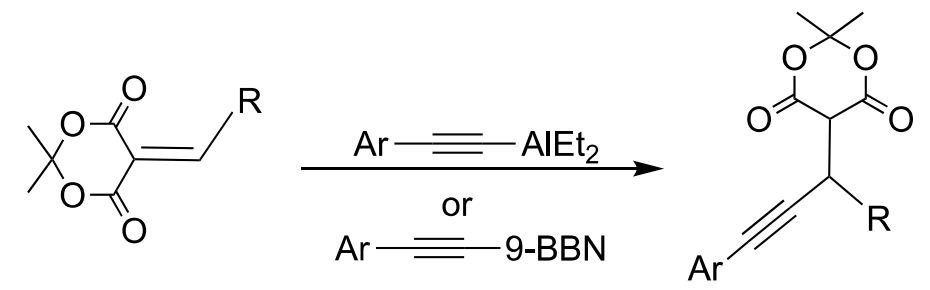

(b)The conjugate addition of in situ generated Cu-alkynylides with Meldrum' s acid derived acceptors

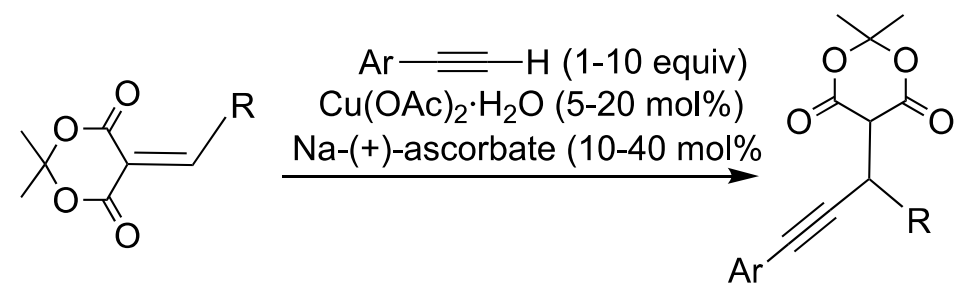

(c) Zinc-mediated conjugate addition of Alkynes to Meldrum's acid derived acceptors

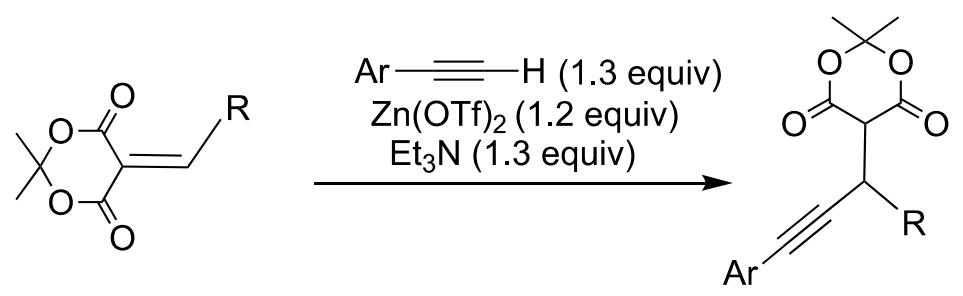

Scheme 1. Reported conjugate additions of alkyne-based metal salts and Meldrum's acid derived acceptors.

The first known method for the conjugate addition of alkynes includes the use of boron ${ }^{8,9}$ or aluminum alkynylides ${ }^{10,11}$ in the presence of $t$-BuMe $\mathrm{SiOTf}^{12-14}$ as an activator under conditions of rigorous exclusion of oxygen and moisture. From a practical point of view, the second method of in situ generated metal alkynylides is attractive, as it can be completed in a single synthetic operation. A series of elegant papers ${ }^{15-19}$ reported the direct conjugate addition of in situ generated Cu-acetylides to Meldrum's acids in the presence of copper acetate, based on $\mathrm{Na}-(+)$-ascorbate as a reductant. This method was optimal only for addition of aylacetylenes to $\gamma$-branched alkylidene acceptors. The third method disclosed ${ }^{20}$ the diastereoselective alkynylation of chiral oxazepanedione acceptors with $\mathrm{Zn}(\mathrm{OTf})_{2}$ and an amine base. The substituents were limited to alkyl groups of Meldrum's acids derived receptors. Hence, the development of a simple, wide substrate and efficient procedure for the synthesis of new 6 -alkynyl Meldrum's acids is still needed. 
In continuation of our efforts toward the development of novel 6 -alkynyl Meldrum's acid compounds, ${ }^{21}$ herein we report the use of $\mathrm{Cu}(\mathrm{OAc})_{2} \cdot \mathrm{H}_{2} \mathrm{O} / \mathrm{Na}$-D-isoascorbate as a catalytic system for the synthesis of 5-(1aryl-3-arylprop-2-yn-1-yl)-2,2-butylidene-1,3-dioxane-4,6-dione derivatives through three-component reactions of an araldehyde, 2,2-butylidene-1,3-dioxane-4,6-dione and an arylacetylene (Scheme 2).

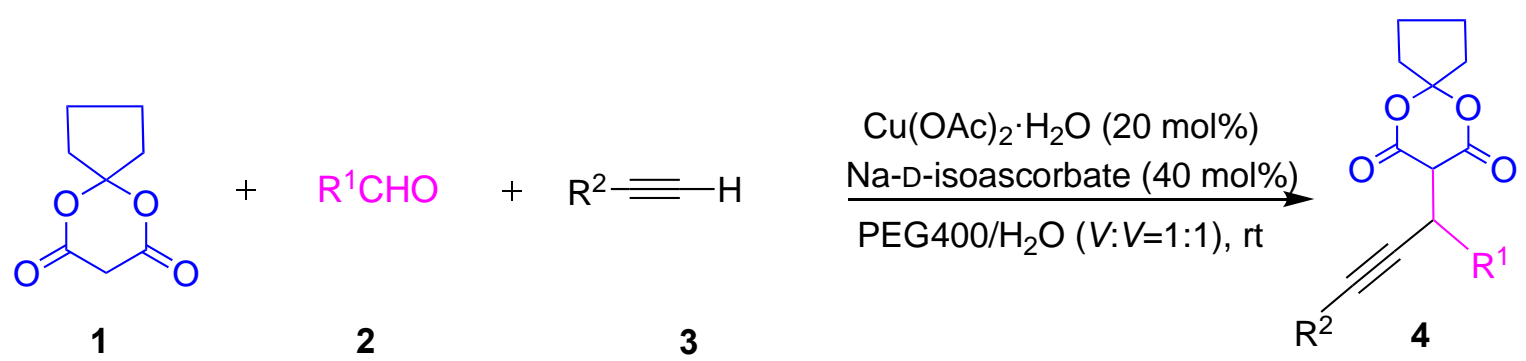

Scheme 2. The three-component synthesis of 5-(1-aryl-3-arylprop-2-yn-1-yl)-2,2-butylidene-1,3-dioxane-4,6diones

\section{Results and Discussion}

For optimizing the reaction conditions, the three-component reaction of 2,2-butylidene-1,3-dioxane-4,6-dione (1), benzaldehyde (2a) and phenylacetylene (3a) was chosen (Table 1). In our initial screening experiments, examination of various copper salts was undertaken. Various copper salts including $\mathrm{CuSO}_{4} \cdot 5 \mathrm{H}_{2} \mathrm{O}$, $\mathrm{Cu}_{2}\left(\mathrm{CO}_{3}\right)(\mathrm{OH})_{2}, \mathrm{Cu}(\mathrm{acac})_{2}, \mathrm{Cu}_{3}\left(\mathrm{PO}_{4}\right)_{2} \cdot 2 \mathrm{H}_{2} \mathrm{O}, \mathrm{Cul}, \mathrm{CuCl}$ and $\mathrm{Cu}(\mathrm{OAc})_{2} \cdot \mathrm{H}_{2} \mathrm{O}$ were examined (Table 1, entries 1-7). Results showed that the yield reached $81 \%$ in the presence of $\mathrm{Cu}(\mathrm{OAC})_{2} \cdot \mathrm{H}_{2} \mathrm{O} / \mathrm{Na}$-D-isoascorbate (Table 1 , entry 7). Encouraged by this result, different reductants such as sodium ascorbate, $\mathrm{Na}_{2} \mathrm{SO}_{3}$ and $\mathrm{NH} \mathrm{H}_{2} \mathrm{OH} \cdot \mathrm{HCl} w e r e$ examined and sodium ascorbate displayed the best efficiency (Table 1, Entries 7-9). We also investigated the effect of reaction time and found that 5.0 hours gave the best result (Table 1, entry 7). Thus, the optimal reaction conditions for 2,2-butylidene-1,3-dioxane-4,6-dione (1,1 mmol), benzaldehyde (2a, $2 \mathrm{mmol})$ and phenylacetylene (3a, 1.5 equiv) involved $\mathrm{Cu}(\mathrm{OAc})_{2} \cdot \mathrm{H}_{2} \mathrm{O}(20 \mathrm{~mol} \%)$ Na-D-isoascorbate $(40 \mathrm{~mol} \%)$ in $\mathrm{PEG} / \mathrm{H}_{2} \mathrm{O}$ $(V: V=1: 1,4 \mathrm{~mL})$, furnishing $4 \mathrm{a}$ in $81 \%$ yield.

Table 1. Optimization of reaction conditions for the synthesis of $4 a^{a}$

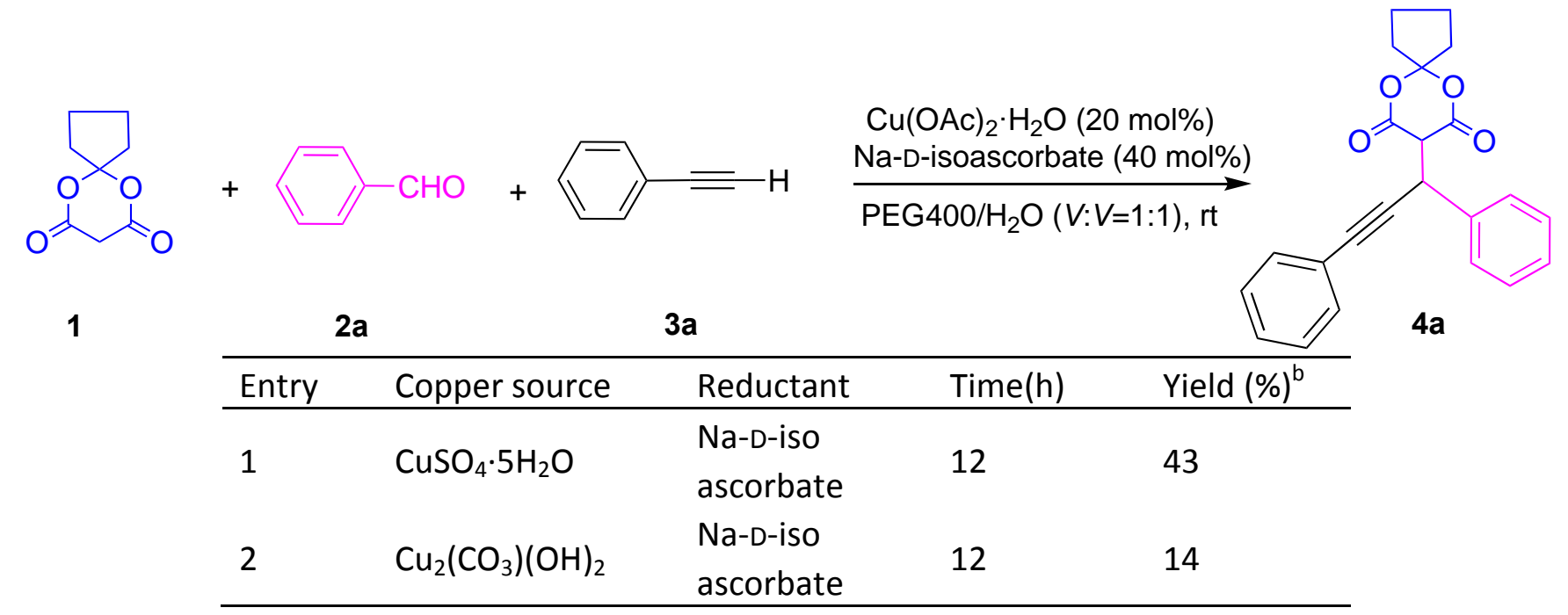


Table 1. Continued

\begin{tabular}{|c|c|c|c|c|}
\hline Entry & Copper source & Reductant & Time(h) & Yield (\%) \\
\hline 3 & $\mathrm{Cu}(\mathrm{acac})_{2}$ & $\begin{array}{l}\text { Na-D-iso } \\
\text { ascorbate }\end{array}$ & 12 & 38 \\
\hline 4 & $\mathrm{Cu}_{3}\left(\mathrm{PO}_{4}\right)_{2} \cdot 2 \mathrm{H}_{2} \mathrm{O}$ & $\begin{array}{l}\text { Na-D-iso } \\
\text { ascorbate }\end{array}$ & 12 & 8 \\
\hline 5 & $\mathrm{CuCl}$ & - & 20 & 0 \\
\hline 6 & Cul & - & 20 & 0 \\
\hline 7 & $\mathrm{Cu}(\mathrm{OAc})_{2} \cdot \mathrm{H}_{2} \mathrm{O}$ & $\begin{array}{l}\mathrm{Na}-\mathrm{D} \text {-iso } \\
\text { ascorbate }\end{array}$ & 5.0 & 81 \\
\hline 8 & $\mathrm{Cu}(\mathrm{OAc})_{2} \cdot \mathrm{H}_{2} \mathrm{O}$ & $\mathrm{NH}_{2} \mathrm{OH} \cdot \mathrm{HCl}$ & 5.0 & 0 \\
\hline 9 & $\mathrm{Cu}(\mathrm{OAc})_{2} \cdot \mathrm{H}_{2} \mathrm{O}$ & $\mathrm{Na}_{2} \mathrm{SO}_{3}$ & 5.0 & 51 \\
\hline 10 & $\mathrm{Cu}(\mathrm{OAc})_{2} \cdot \mathrm{H}_{2} \mathrm{O}$ & $\begin{array}{l}\mathrm{Na} \text {-D-iso } \\
\text { ascorbate }\end{array}$ & 4.0 & 70 \\
\hline 11 & $\mathrm{Cu}(\mathrm{OAc})_{2} \cdot \mathrm{H}_{2} \mathrm{O}$ & $\begin{array}{l}\mathrm{Na}-\mathrm{D}-\mathrm{iso} \\
\text { ascorbate }\end{array}$ & 6.0 & 81 \\
\hline
\end{tabular}

a Reaction conditions: 2,2-butylidene-1,3-dioxane-4,6-dione (1, $1 \mathrm{mmol})$, benzaldehyde ( $2 \mathrm{a}, 2 \mathrm{mmol}$ ), Cu salt ( $20 \mathrm{~mol} \%), \mathrm{PhC} \equiv \mathrm{CH}$ (1.5 equiv), reductant (40 mol\%), PEG400/ $\mathrm{H}_{2} \mathrm{O}(\mathrm{V}: \mathrm{V}=1: 1)\left(4 \mathrm{~mL}, \mathrm{rt} ;{ }^{\mathrm{b}}\right.$ Isolated yield.

Using the optimized conditions, a number of substrates were investigated (Table 2). A variety of substituents, electron-rich and -poor aromatic groups, heteroaromatic (Table 2, entries 1-7), branched (Table 2, entry 11), and unbranched (Table 2 entries 9-11) aliphatic, as well as alkenes (Table 2, entry 8), can be tolerated on the aldehydes. 4-Chlorophenylacetylene also participated in this reaction effectively (Table 2 entries 12, 13).

Table 2. Synthesis of products 4 promoted by $\mathrm{Cu}(\mathrm{OAc})_{2} \cdot \mathrm{H}_{2} \mathrm{O} / \mathrm{Na}$-D-isoascorbate ${ }^{\mathrm{a}}$

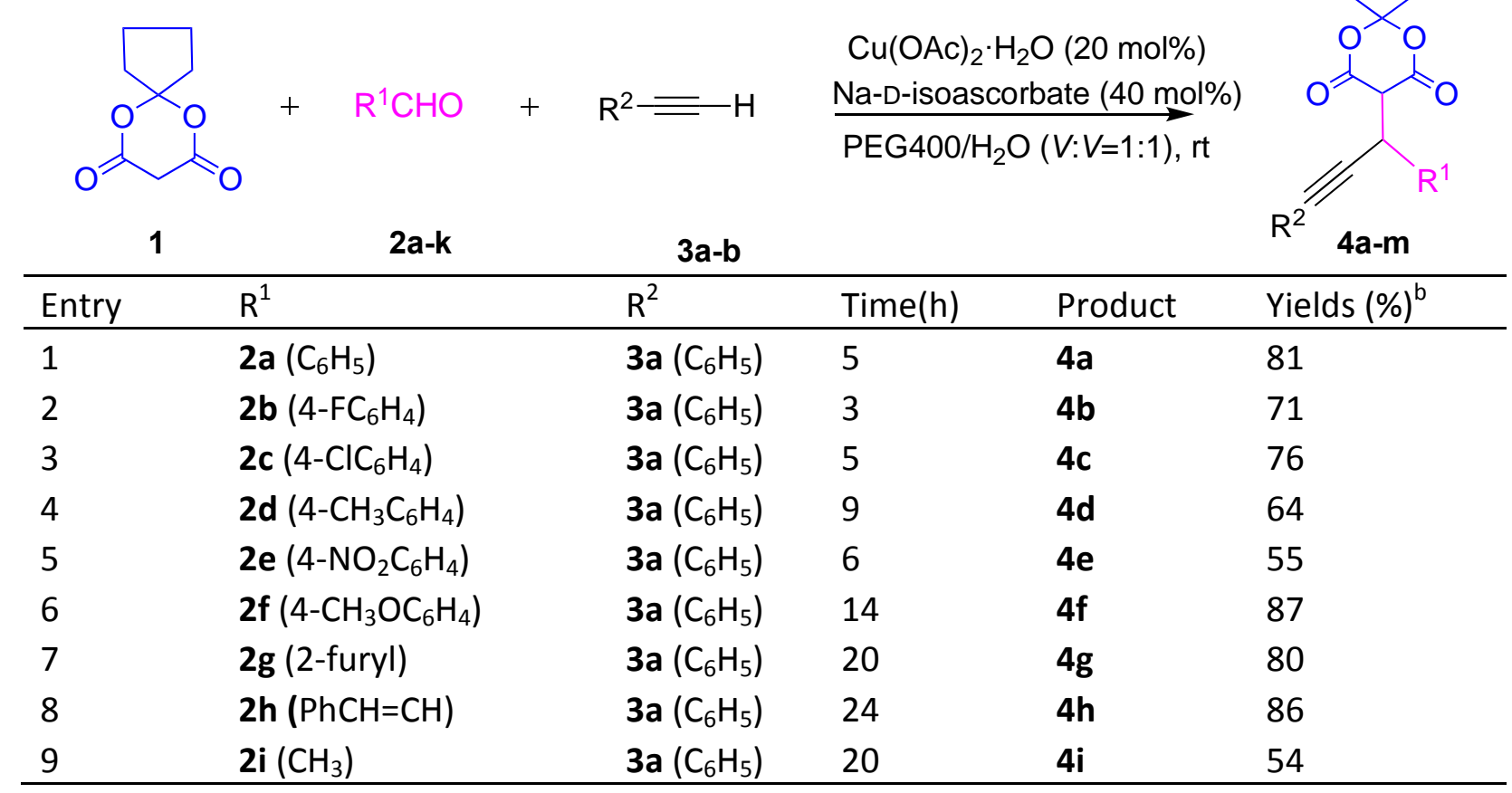


Table 2. Continued

\begin{tabular}{llllll}
\hline Entry & $\mathrm{R}^{1}$ & $\mathrm{R}^{2}$ & Time(h) & Product & Yields (\%) \\
\hline 10 & $\mathbf{2} \mathbf{j}\left(\mathrm{CH}_{3}\left(\mathrm{CH}_{2}\right)_{2}\right)$ & $\mathbf{3 a}\left(\mathrm{C}_{6} \mathrm{H}_{5}\right)$ & 20 & $\mathbf{4 j}$ & 67 \\
11 & $\left.\left.\mathbf{2}\left(\mathrm{CH}_{3}\right)_{2} \mathrm{CH}\right)\right)$ & $\mathbf{3 a}\left(\mathrm{C}_{6} \mathrm{H}_{5}\right)$ & 20 & $\mathbf{4 k}$ & 64 \\
12 & $\mathbf{2 a}\left(\mathrm{C}_{6} \mathrm{H}_{5}\right)$ & $\mathbf{3 b}(4-$ & 8 & $\mathbf{4 l}$ & 72 \\
& & $\left.\mathrm{ClC}_{6} \mathrm{H}_{4}\right)$ & & & \\
13 & $\mathbf{2 j}\left(\mathrm{CH}_{3}\left(\mathrm{CH}_{2}\right)_{2}\right)$ & $\mathbf{3 b}(4-$ & 16 & $\mathbf{4 m}$ & 68 \\
\hline
\end{tabular}

${ }^{\mathrm{a}}$ Reaction conditions: 2,2-butylidene-1,3-dioxane-4,6-dione (1, $\left.1 \mathrm{mmol}\right)$, aldehyde $(2,2 \mathrm{mmol})$, $\mathrm{ArC} \equiv \mathrm{CH}$ (1.5 equiv), $\mathrm{Cu}(\mathrm{OAc})_{2} \cdot \mathrm{H}_{2} \mathrm{O}(20 \mathrm{~mol} \%), \mathrm{Na}-\mathrm{D}$-isoascorbate $(40 \mathrm{~mol} \%), \mathrm{PEG} 400 / \mathrm{H}_{2} \mathrm{O}$ $(V: V=1: 1)(4 \mathrm{~mL}), \mathrm{rt} ;{ }^{\mathrm{b}}$ Isolated yield

In order to gain further information on the intermediate formation of the phenylethynyl-Cu(I) 6, After reduction of $\mathrm{Cu}(\mathrm{OAc})_{2} \cdot \mathrm{H}_{2} \mathrm{O}(20 \mathrm{~mol} \%)$ with Na-D-isoascorbate $(40 \mathrm{~mol} \%)$ in $\mathrm{PEG} 400 / \mathrm{H}_{2} \mathrm{O}$, phenylacetylene (1.5 equiv) was added. The resulting mixture was stirred for $5.0 \mathrm{~h}$, then the mixture was extracted with $\mathrm{CH}_{2} \mathrm{Cl}_{2}$, dried over $\mathrm{Na}_{2} \mathrm{SO}_{4}$, concentrated under reduced pressure. The yellow residue obtained was washed with absolute EtOH and dried in a vacuum. The yellow powder was subjected to infra-red and mass spectroscopic analysis. In the high-resolution MALDI-TOF mass spectrum, the major peak corresponded to ( $\mathrm{PhC} \equiv \mathrm{CCU}+\mathrm{Na}$ ) $\mathrm{m} / z$ 187.0. The stretching frequencies of the $C \equiv C$ bond decreased from $2120 \mathrm{~cm}^{-1}$ for phenylacetylene to 1929 $\mathrm{cm}^{-1}$ for the copper alkynylide. Based on the above results, a reasonable mechanism for the one-pot synthesis of 5-(1-phenyl-3-phenylprop-2-yn-1-yl)-2,2-butylidene-1,3-dioxane-4,6-dione 4a is depicted in Scheme 3. The terminal $\mathrm{C}-\mathrm{H}$ of phenylacetylene $3 \mathrm{a}$ is activated by $\mathrm{Cu}(\mathrm{I})$ prepared from $\mathrm{Cu}(\mathrm{OAc})_{2} \cdot \mathrm{H}_{2} \mathrm{O}$ in the presence of Na-Disoascorbate, and thence phenylethynyl-Cu(I) $\mathbf{6}$ is formed. Subsequently, the product $\mathbf{4 a}$ is obtained by the conjugate addition reaction of phenylethynyl- $\mathrm{Cu}(\mathrm{I}) 6$ and 5-phenylmethylene-2,2-butylidene-1,3-dioxane-4,6dione 5 (resulting from a Knoevenagel reaction of the benzaldehyde and 2,2-butylidene-1,3-dioxane-4,6-dione 1).

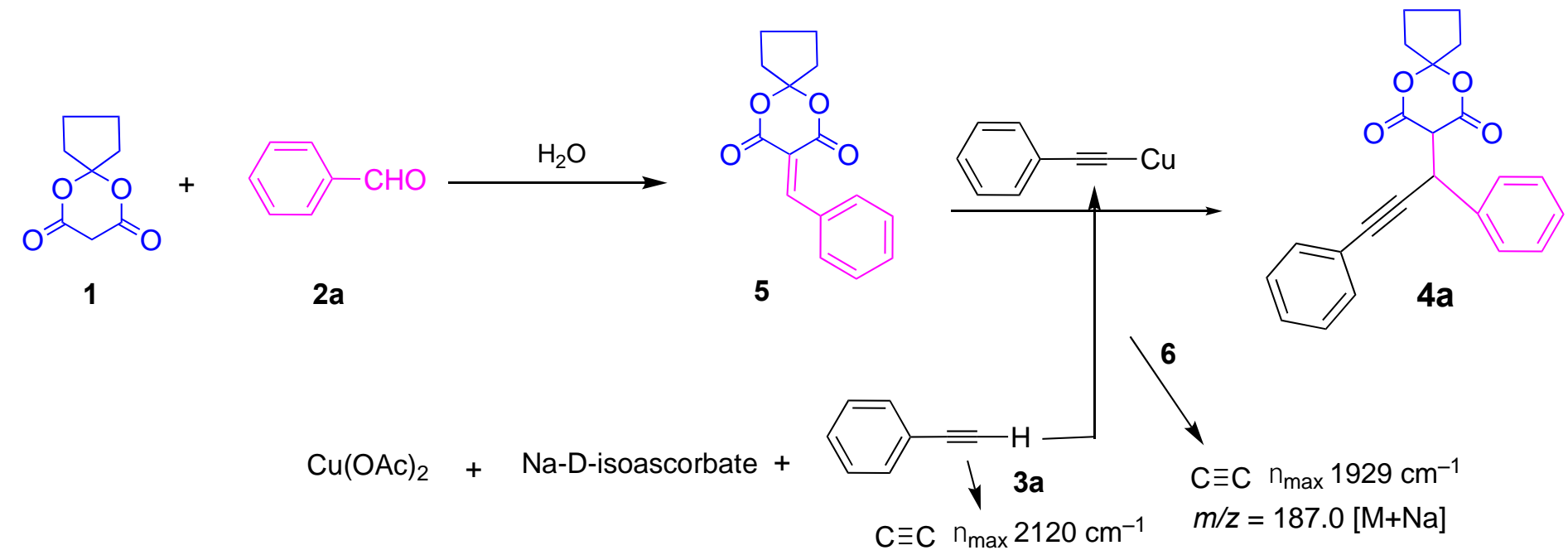

Scheme 3. Proposed mechanism for the formation of $\mathbf{4 a .}$ 


\section{Conclusions}

A three-component synthetic procedure of 5-(1-phenyl-3-phenylprop-2-yn-1-yl)-2,2-butylidene-1,3-dioxane4,6-dione derivatives catalyzed by a combination of $\mathrm{Cu}(\mathrm{OAC})_{2} \cdot\left(\mathrm{H}_{2} \mathrm{O}\right)$ and $\mathrm{Na}$-D-isoascorbate, has been developed. The operation and work-up procedures were very simple and no column chrommatography purification was

needed. This provides an effective method for the synthesis of new $\beta$-arylalkynyl Meldrum's acid analogues.

\section{Experimental Section}

General. 2,2-Butylidene-1,3-dioxane-4,6-dione was prepared according to the literature. ${ }^{22-24}$ The other chemicals were purchased from Aladdin, Aldrich and Fluka Chemical Companies and used without further purification. Melting points were measured on XT-4 digital micro melting point apparatus and are uncorrected. ${ }^{1} \mathrm{H}$ NMR spectra were recorded on a BRUKER AVANCE $400 \mathrm{MHz}$ spectrometer using $\mathrm{CDCl}_{3}$ as the solvent and TMS as the internal standard. ${ }^{13} \mathrm{C}$ NMR data were collected on a BRUKER AVANCE $100 \mathrm{MHz}$ instrument with $\mathrm{CDCl}_{3}$ as the solvent and TMS as the internal standard. The analytical mass spectrometry was performed on an Agilent LC-MSD Trap VL Apparatus.

Typical one-pot procedure for the synthesis of 4a. To a $25 \mathrm{~mL}$ tube equipped with a stirring bar were added PEG400/ $\mathrm{H}_{2} \mathrm{O}(\mathrm{V}: \mathrm{V}=1: 1,4.0 \mathrm{~mL}), \mathrm{Cu}(\mathrm{OAc})_{2} \cdot \mathrm{H}_{2} \mathrm{O}(0.2 \mathrm{mmol}, 20 \mathrm{~mol} \%)$, phenylacetylene (3a, $\left.1.5 \mathrm{mmol}\right), \mathrm{Na}-\mathrm{D}-\mathrm{iso}$ ascorbate (0.4 mmol, 40 mol\%), 2,2-butylidene-1,3-dioxane-4,6-dione (1, $1 \mathrm{mmol})$ and benzaldehyde (2a, 2 mmol). The reaction mixture was stirred vigorously for $5.0 \mathrm{~h}$, treated with $\mathrm{CH}_{2} \mathrm{Cl}_{2}$ and sat aq. $\mathrm{NH}_{4} \mathrm{Cl}$ soln. The organic layer was separated and the water phase was extracted twice with $\mathrm{CH}_{2} \mathrm{Cl}_{2}$. The combined organic layers were dried over $\mathrm{Na}_{2} \mathrm{SO}_{4}$ and concentrated under reduced pressure. The residue was purified by recrytallization from absolute EtOH to afford the pure product.

5-(1,3-Diphenylprop-2-yn-1-yl)-2,2-butylidene-1,3-dioxane-4,6-dione (4a). White solid, mp 156-158 ${ }^{\circ} \mathrm{C}$ (Yield: 81\%). ${ }^{1} \mathrm{H}$ NMR (400 MHz, $\left.\mathrm{CDCl}_{3}\right): \delta(\mathrm{ppm}): 1.78-1.93\left(4 \mathrm{H}, \mathrm{m}, 2 \mathrm{CH}_{2}\right), 2.09-2.19\left(4 \mathrm{H}, \mathrm{m}, 2 \mathrm{CH}_{2}\right), 4.01\left(1 \mathrm{H}, \mathrm{d},{ }^{3} J_{H H}\right.$ $2.8 \mathrm{~Hz}, \mathrm{CH}), 5.11\left(1 \mathrm{H}, \mathrm{d},{ }^{3} J_{H H} 2.8 \mathrm{~Hz}, \mathrm{CH}\right), 7.27-7.38(6 \mathrm{H}, \mathrm{m}, \mathrm{HAr}), 7.45-7.51(2 \mathrm{H}, \mathrm{m}, \mathrm{HAr}), 7.65\left(2 \mathrm{H}, \mathrm{d},{ }^{3} J_{H H} 7.2 \mathrm{~Hz}\right.$, $2 \mathrm{CH}, \mathrm{HAr}) .{ }^{13} \mathrm{C} N M R\left(100 \mathrm{MHz}, \mathrm{CDCl}_{3}\right): \delta(\mathrm{ppm}): 22.6,24.2,35.9,38.5,38.9,53.9,85.1,86.3,114.2,122.9$, 127.7, 128.2, 128.3, 128.5, 128.8, 131.9, 137.3, 163.0, 164.0. HRMS (m/z): $[\mathrm{M}+\mathrm{Na}]^{+}$calcd for $\mathrm{C}_{23} \mathrm{H}_{20} \mathrm{NaO}_{4}$, 383.1259; found, 383.1247.

5-[1-(4-Fluorophenyl)-3-phenylprop-2-yn-1-yl]-2,2-butylidene-1,3-dioxane-4,6-dione (4b). White solid, mp 125-127 ${ }^{\circ} \mathrm{C}$ (Yield: 71\%). ${ }^{1} \mathrm{H}$ NMR (400 MHz, CDCl $): \delta(p p m): 1.79-1.93\left(4 \mathrm{H}, \mathrm{m}, 2 \mathrm{CH}_{2}\right), 2.09-2.20\left(4 \mathrm{H}, \mathrm{m}, 2 \mathrm{CH}_{2}\right)$, $3.98\left(1 \mathrm{H}, \mathrm{d},{ }^{3} J_{H H} 2.8 \mathrm{~Hz}, \mathrm{CH}\right), 5.10\left(1 \mathrm{H}, \mathrm{d},{ }^{3} J_{H H} 2.8 \mathrm{~Hz}, \mathrm{CH}\right), 7.04\left(2 \mathrm{H}, \mathrm{t},{ }^{3} J_{H H} 8.4 \mathrm{~Hz}, \mathrm{HAr}\right), 7.28-7.34(3 \mathrm{H}, \mathrm{m}, \mathrm{HAr})$, $7.47\left(2 \mathrm{H}, \mathrm{dd},{ }^{4} J_{H F} 2.0 \mathrm{~Hz},{ }^{3} \mathrm{~J}_{\mathrm{HH}} 5.2 \mathrm{~Hz}, \mathrm{HAr}\right), 7.64\left(2 \mathrm{H}, \mathrm{dd},{ }^{3} J_{\mathrm{HF}} 8.4 \mathrm{~Hz},{ }^{3} \mathrm{~J}_{H H} 5.2 \mathrm{~Hz}, \mathrm{HAr}\right) .{ }^{13} \mathrm{C} \mathrm{NMR}\left(100 \mathrm{MHz} \mathrm{CDCl}_{3}\right)$ : $\delta$ (ppm): 22.5, 24.4, 35.2, 38.5, 38.9, 53.8, 85.1, 86.2, 114.3, 115.2, 115.4, 122.7, 128.3, 128.4, $130.7\left(\mathrm{~d},{ }^{2} J_{C F} 8.0\right.$ $\mathrm{Hz}), 131.9,132.8\left(\mathrm{~d},{ }^{3} J_{C F} 3.1 \mathrm{~Hz}\right), 161.0\left(\mathrm{~d},{ }^{1} J_{C F} 245.1 \mathrm{~Hz}\right), 163.1,163.7 . \mathrm{HRMS}(\mathrm{m} / \mathrm{z}):[\mathrm{M}+\mathrm{Na}]^{+}$calcd for $\mathrm{C}_{23} \mathrm{H}_{19} \mathrm{FNaO}_{4}, 401.1165$; found, 401.1182.

5-[1-(4-Chlorophenyl)-3-phenylprop-2-yn-1-yl]-2,2-butylidene-1,3-dioxane-4,6-dione (4c). White solid, mp 126-128 ${ }^{\circ} \mathrm{C}$ (Yield: 76\%). ${ }^{1} \mathrm{H}$ NMR (400 MHz, CDCl $): \delta(p p m): 1.79-1.93\left(4 \mathrm{H}, \mathrm{m}, 2 \mathrm{CH}_{2}\right), 2.09-2.21\left(4 \mathrm{H}, \mathrm{m}, 2 \mathrm{CH}_{2}\right)$, $3.99\left(1 \mathrm{H}, \mathrm{d},{ }^{3} \mathrm{~J}_{H H} 2.8 \mathrm{~Hz}, \mathrm{CH}\right), 5.08\left(1 \mathrm{H}, \mathrm{d},{ }^{3} J_{H H} 2.8 \mathrm{~Hz}, \mathrm{CH}\right), 7.28-7.33(5 \mathrm{H}, \mathrm{m}, \mathrm{HAr}), 7.45-7.49(2 \mathrm{H}, \mathrm{m}, \mathrm{HAr}), 7.61$ $\left(2 \mathrm{H}, \mathrm{d},{ }^{3} \mathrm{~J}_{H H} 8.4 \mathrm{~Hz}, 2 \mathrm{CH}, \mathrm{HAr}\right) .{ }^{13} \mathrm{C} N M R\left(100 \mathrm{MHz}, \mathrm{CDCl}_{3}\right): \delta(\mathrm{ppm}): 22.5,24.2,35.3,38.5,38.9,53.7,85.3,85.9$, 
$114.3,122.6,128.3,128.5,128.6,130.4,131.9,133.7,135.7,163.0,163.7 . ~ H R M S ~(m / z): ~[M+N a]^{+}$calcd for $\mathrm{C}_{23} \mathrm{H}_{19} \mathrm{ClNaO}_{4}, 417.0870$; found, 417.0882 .

5-[1-(4-Methylphenyl)-3-phenylprop-2-yn-1-yl]-2,2-butylidene-1,3-dioxane-4,6-dione (4d). White solid, mp 139-141 ${ }^{\circ} \mathrm{C}$ (Yield: $\left.64 \%\right) .{ }^{1} \mathrm{H}$ NMR (400 MHz, $\left.\mathrm{CDCl}_{3}\right): \delta(\mathrm{ppm}):$ 1.77-1.91 $\left(4 \mathrm{H}, \mathrm{m}, 2 \mathrm{CH}_{2}\right), 2.07-2.19\left(4 \mathrm{H}, \mathrm{m}, 2 \mathrm{CH}_{2}\right)$, $2.33\left(3 \mathrm{H}, \mathrm{s}, \mathrm{CH}_{3}\right), 3.99\left(1 \mathrm{H}, \mathrm{d},{ }^{3} J_{H H} 2.8 \mathrm{~Hz}, \mathrm{CH}\right), 5.08\left(1 \mathrm{H}, \mathrm{d},{ }^{3} J_{H H} 2.8 \mathrm{~Hz}, \mathrm{CH}\right), 7.16\left(2 \mathrm{H}, \mathrm{d},{ }^{3} J_{H H} 8.0 \mathrm{~Hz}, 2 \mathrm{CH}, \mathrm{HAr}\right)$, 7.26-7.32 (3H, m, HAr), 7.44-7.49 (2H, m, HAr), $7.54\left(2 \mathrm{H}, \mathrm{d},{ }^{3} \mathrm{~J}_{H H} 8.0 \mathrm{~Hz}, 2 \mathrm{CH}, \mathrm{HAr}\right) .{ }^{13} \mathrm{C} \mathrm{NMR}\left(100 \mathrm{MHz}^{\mathrm{C} C D C l}\right)_{3}: \delta$ (ppm): 21.1, 22.5, 24.2, 35.6, 38.5, 38.9, 53.9, 84.9, 86.6, 114.2, 123.0, 128.2, 128.3, 128.7, 129.2, 131.9, 134.3, 137.4, 163.1, 164.1; HRMS (m/z): [M+Na] $]^{+}$calcd for $\mathrm{C}_{24} \mathrm{H}_{22} \mathrm{NaO}_{4}, 397.1416$; found, 397.1408.

5-[1-(4-Nitrophenyl)-3-phenylprop-2-yn-1-yl]-2,2-butylidene-1,3-dioxane-4,6-dione (4e). White solid, mp 136-138 ${ }^{\circ} \mathrm{C}$ (Yield: $\left.55 \%\right) .{ }^{1} \mathrm{H}$ NMR $\left(400 \mathrm{MHz} \mathrm{CDCl}_{3}\right): \delta(\mathrm{ppm}): 1.81-1.96\left(4 \mathrm{H}, \mathrm{m}, 2 \mathrm{CH}_{2}\right), 2.12-2.25\left(4 \mathrm{H}, \mathrm{m}, 2 \mathrm{CH}_{2}\right)$, 4.05(1H, d, $\left.{ }^{3} J_{H H} 2.8 \mathrm{~Hz}, \mathrm{CH}\right), 5.20\left(1 \mathrm{H}, \mathrm{d},{ }^{3} J_{H H} 2.8 \mathrm{~Hz}, \mathrm{CH}\right), 7.30-7.37(3 \mathrm{H}, \mathrm{m}, \mathrm{HAr}), 7.47-7.52(2 \mathrm{H}, \mathrm{m}, \mathrm{HAr})$, $7.87\left(2 \mathrm{H}, \mathrm{d},{ }^{3} \mathrm{~J}_{\mathrm{HH}} 8.8 \mathrm{~Hz}, 2 \mathrm{CH}, \mathrm{HAr}\right), 8.22\left(2 \mathrm{H}, \mathrm{d},{ }^{3} \mathrm{~J}_{H H} 8.8 \mathrm{~Hz}, 2 \mathrm{CH}, \mathrm{HAr}\right) .{ }^{13} \mathrm{C} \mathrm{NMR}(100 \mathrm{MHz}, \mathrm{CDCl}): \delta(\mathrm{ppm}): 22.5$, 24.3, 35.6, 38.6, 38.9, 53.6, 84.5, 85.8, 114.5, 123.6, 128.4, 128.8, 130.1, 132.0, 144.4, 147.4, $162.9,164.1$. HRMS $(m / z):[\mathrm{M}+\mathrm{Na}]^{+}$calcd for $\mathrm{C}_{23} \mathrm{H}_{19} \mathrm{NNaO}_{6}, 428.1110$; found, 428.1116 .

5-[1-(4-Methoxylphenyl)-3-phenylprop-2-yn-1-yl]-2,2-butylidene-1,3-dioxane-4,6-dione (4f). Light yellow solid, mp $125-127{ }^{\circ} \mathrm{C}$ (Yield: 87\%). ${ }^{1} \mathrm{H}$ NMR $\left(400 \mathrm{MHz}, \mathrm{CDCl}_{3}\right): \delta(\mathrm{ppm}): 1.78-1.92\left(4 \mathrm{H}, \mathrm{m}, 2 \mathrm{CH}_{2}\right), 2.08-2.20(4 \mathrm{H}$, $\left.\mathrm{m}, 2 \mathrm{CH}_{2}\right), 3.80\left(3 \mathrm{H}, \mathrm{s}, \mathrm{CH}_{3} \mathrm{O}\right), 3.98\left(1 \mathrm{H}, \mathrm{d},{ }^{3} \mathrm{~J}_{H H} 2.8 \mathrm{~Hz}, \mathrm{CH}\right), 5.06\left(1 \mathrm{H}, \mathrm{d},{ }^{3} \mathrm{~J}_{H H} 2.8 \mathrm{~Hz}, \mathrm{CH}\right), 6.88\left(2 \mathrm{H}, \mathrm{d},{ }^{3} \mathrm{~J}_{H H} 8.4 \mathrm{~Hz}\right.$, 2CH, HAr), 7.28-7.31 (3H, m, HAr), 7.44-7.49 (2H, m, HAr), $7.58\left(2 \mathrm{H}, \mathrm{d},{ }^{3} \mathrm{~J}_{H H} 8.4 \mathrm{~Hz}, 2 \mathrm{CH}, \mathrm{HAr}\right) .{ }^{13} \mathrm{C} \mathrm{NMR}(100$ $\left.\mathrm{MHz}_{1} \mathrm{CDCl}_{3}\right): \delta$ (ppm): 22.6, 24.2, 35.2, 38.6, 38.9, 53.9, 55.3, 84.8, 86.8, 113.8, 114.2, 123.0, 128.2, 128.3, 129.1, 130.1, 131.9, 159.1, 163.2, 163.9. HRMS $(\mathrm{m} / \mathrm{z})$ : $[\mathrm{M}+\mathrm{Na}]^{+}$calcd for $\mathrm{C}_{24} \mathrm{H}_{22} \mathrm{NaO}_{5}, 413.1365$; found, 413.1381.

5-(1-(Furan-2-yl)-3-phenylprop-2-yn-1-yl)-2,2-butylidene-1,3-dioxane-4,6-dione (4g). Off-white solid, mp 130$131{ }^{\circ} \mathrm{C}$ (Yield: 80\%). ${ }^{1} \mathrm{H}$ NMR $\left(400 \mathrm{MHz}, \mathrm{CDCl}_{3}\right): \delta(\mathrm{ppm}): 1.82-1.96\left(4 \mathrm{H}, \mathrm{m}, 2 \mathrm{CH}_{2}\right), 2.17-2.28\left(4 \mathrm{H}, \mathrm{m}, 2 \mathrm{CH}_{2}\right), 4.22$ $\left(1 \mathrm{H}, \mathrm{d},{ }^{3} J_{H H} 2.8 \mathrm{~Hz}, \mathrm{CH}\right), 5.11\left(1 \mathrm{H}, \mathrm{d},{ }^{3} J_{H H} 2.8 \mathrm{~Hz}, \mathrm{CH}\right), 6.38\left(1 \mathrm{H}, \mathrm{dd},{ }^{3} J_{H H} 3.2,2.0 \mathrm{~Hz}, \mathrm{CH}, \mathrm{H}_{\text {furan }}\right), 6.55\left(1 \mathrm{H}, \mathrm{dd},{ }^{3} J_{H H}\right.$ 3.2, $\left.0.8 \mathrm{~Hz}, \mathrm{CH}, \mathrm{H}_{\text {furan }}\right), 7.27-7.32(3 \mathrm{H}, \mathrm{m}, \mathrm{HAr}), 7.33\left(1 \mathrm{H}, \mathrm{t},{ }^{3} \mathrm{~J}_{H H} 2.0,0.8 \mathrm{~Hz}, \mathrm{CH}, \mathrm{H}_{\text {furan }}\right), 7.43-7.47(2 \mathrm{H}, \mathrm{m}, \mathrm{HAr})$. ${ }^{13} \mathrm{C} \mathrm{NMR}\left(100 \mathrm{MHz}, \mathrm{CDCl}_{3}\right): \delta$ (ppm): 22.6, 24.3, 30.4, 38.6, 38.9, 50.6, 83.9, 84.1, 108.4, 111.0, 114.3, 122.5, 128.2, 128.5, 132.0, 141.8, 150.1, 162.7, 163.6. HRMS $(\mathrm{m} / \mathrm{z}):[\mathrm{M}+\mathrm{Na}]^{+}$calcd for $\mathrm{C}_{21} \mathrm{H}_{18} \mathrm{NaO}_{5}, 373.1052 ;$ found, 373.1064 .

(E)-5-[3-phenyl-1-(phenylethynyl)prop-2-en-1-yl]-2,2-butylidene-1,3-dioxane-4,6-dione (4h). White solid, mp 135-137 ${ }^{\circ} \mathrm{C}$ (Yield: 86\%). ${ }^{1} \mathrm{H}$ NMR (400 MHz, CDCl 3 ): $\delta(p p m): 1.81-1.96\left(4 \mathrm{H}, \mathrm{m}, 2 \mathrm{CH}_{2}\right), 2.18-2.27\left(4 \mathrm{H}, \mathrm{m}, 2 \mathrm{CH}_{2}\right)$, $3.91\left(1 \mathrm{H}, \mathrm{d},{ }^{3} \mathrm{~J}_{H H} 2.8 \mathrm{~Hz}, \mathrm{CH}\right), 4.54\left(1 \mathrm{H}, \mathrm{ddd},{ }^{3} \mathrm{~J}_{H H} 2.8,3.6 \mathrm{~Hz},{ }^{4} J_{H H} 0.8 \mathrm{~Hz}, \mathrm{CH}\right), 6.51\left(1 \mathrm{H}, \mathrm{dd},{ }^{3} J_{H H} 15.6,8.0 \mathrm{~Hz}, \mathrm{CH}\right.$, $\left.\mathrm{H}_{\mathrm{C}=\mathrm{C}}\right), 6.81\left(1 \mathrm{H}, \mathrm{d},{ }^{3} \mathrm{~J}_{\mathrm{HH}} 15.6 \mathrm{~Hz}, \mathrm{CH}, \mathrm{H}_{\mathrm{C}=\mathrm{C}}\right), 7.22-7.33(6 \mathrm{H}, \mathrm{m}, \mathrm{HAr}), 7.41-7.47(4 \mathrm{H}, \mathrm{m}, \mathrm{HAr}) .{ }^{13} \mathrm{C} \mathrm{NMR}(100 \mathrm{MHz}$, $\left.\mathrm{CDCl}_{3}\right) \delta$ (ppm): 22.6, 24.3, 33.7, 38.5, 39.0, 52.6, 84.2, 86.4, 114.3, 122.9, 124.8, 126.7, 128.0, 128.2, 128.3, 128.6, 131.9, 134.1, 136.3, 163.4, 163.5. HRMS $(\mathrm{m} / \mathrm{z})$ : $[\mathrm{M}+\mathrm{Na}]^{+}$calcd for $\mathrm{C}_{25} \mathrm{H}_{22} \mathrm{NaO}_{4}, 409.1416$; found, 409.1421.

5-(1-Methyl-3-phenylprop-2-yn-1-yl)-2,2-butylidene-1,3-dioxane-4,6-dione (4i). White solid, mp125-126 ${ }^{\circ} \mathrm{C}$ (Yield: 54\%). ${ }^{1} \mathrm{H}$ NMR $\left(400 \mathrm{MHz} \mathrm{CDCl}_{3}\right): \delta(\mathrm{ppm}): 1.55\left(3 \mathrm{H}, \mathrm{d},{ }^{3} \mathrm{~J}_{\mathrm{HH}} 7.2 \mathrm{~Hz}, \mathrm{CH}_{3}\right), 1.82-1.96\left(4 \mathrm{H}, \mathrm{m}, 2 \mathrm{CH}_{2}\right), 2.17-$ $2.27\left(4 \mathrm{H}, \mathrm{m}, 2 \mathrm{CH}_{2}\right), 3.72\left(1 \mathrm{H}, \mathrm{d},{ }^{3} \mathrm{~J}_{H H} 3.2 \mathrm{~Hz}, \mathrm{CH}\right), 3.79\left(1 \mathrm{H}, \mathrm{ddd},{ }^{3} \mathrm{~J}_{H H} 2.8,7.2 \mathrm{~Hz}, \mathrm{CH}\right), 7.27-7.31(3 \mathrm{H}, \mathrm{m}, \mathrm{HAr})$, 7.38-7.42 (2H, m, HAr). $\left.{ }^{13} \mathrm{C} \mathrm{NMR} \mathrm{(100} \mathrm{MHz,} \mathrm{CDCl} 3\right): \delta$ (ppm): 17.7, 22.6, 24.3, 25.0, 38.4, 39.1, 51.9, 82.1, 89.4, 114.1, 123.1, 128.0, 128.1, 131.8, 163.6, 163.8; $\operatorname{HRMS}(\mathrm{m} / \mathrm{z}):[\mathrm{M}+\mathrm{Na}]^{+}$calcd for $\mathrm{C}_{18} \mathrm{H}_{18} \mathrm{NaO}_{4}, 321.1103$; found, 321.1095.

5-(1-Propyl-3-phenylprop-2-yn-1-yl)-2,2-butylidene-1,3-dioxane-4,6-dione (4j). White solid, $\mathrm{mp} 105-106{ }^{\circ} \mathrm{C}$ (Yield: 67\%). ${ }^{1} \mathrm{H}$ NMR (400 MHz, $\left.\mathrm{CDCl}_{3}\right): \delta(\mathrm{ppm}): 0.99\left(3 \mathrm{H}, \mathrm{t},{ }^{3} \mathrm{~J}_{\mathrm{HH}} 7.2 \mathrm{~Hz}, \mathrm{CH}_{3}\right), 1.44-1.61\left(2 \mathrm{H}, \mathrm{m}, \mathrm{H}_{\mathrm{CH} 2}\right), 1.64-$ $1.75\left(1 \mathrm{H}, \mathrm{m}, \mathrm{H}_{\mathrm{CH} 2}\right), 1.81-1.96\left(4 \mathrm{H}, \mathrm{m}, 2 \mathrm{CH}_{2}\right), 2.10-2.16\left(1 \mathrm{H}, \mathrm{m}, \mathrm{H}_{\mathrm{CH} 2}\right), 2.18-2.27\left(4 \mathrm{H}, \mathrm{m}, 2 \mathrm{CH}_{2}\right), 3.62\left(1 \mathrm{H}, \mathrm{ddd},{ }^{3} \mathrm{~J}_{H H}\right.$ 
2.8, 4.4, 7.2 Hz, CH), 3.71(d, $\left.{ }^{3} \mathrm{~J}_{H H} 2.8 \mathrm{~Hz}, 1 \mathrm{H}\right), 7.25-7.28(3 \mathrm{H}, \mathrm{m}, \mathrm{HAr}), 7.38-7.42(2 \mathrm{H}, \mathrm{m}, \mathrm{HAr}) .{ }^{13} \mathrm{C} \mathrm{NMR}(100 \mathrm{MHz}$, $\mathrm{CDCl}_{3}$ ): $\delta$ (ppm): 13.6, 21.3, 22.6, 24.3, 30.7, 34.1, 38.5, 39.0, 51.2, 83.1, 88.3, 114.1, 123.2, 128.0, 128.1, 131.8, 163.8, 164.1. HRMS (m/z): [M+Na] $]^{+}$calcd for $\mathrm{C}_{20} \mathrm{H}_{22} \mathrm{NaO}_{4}, 349.1416$; found, 349.1429.

5-(1-Isopropyl-3-phenylprop-2-yn-1-yl)-2,2-butylidene-1,3-dioxane-4,6-dione (4k). White solid, mp 106-107 ${ }^{\circ} \mathrm{C}$ (Yield: 64\%). ${ }^{1} \mathrm{H}$ NMR $\left(400 \mathrm{MHz}, \mathrm{CDCl}_{3}\right): \delta(\mathrm{ppm}): 1.01\left(3 \mathrm{H}, \mathrm{d},{ }^{3} \mathrm{~J}_{H H} 6.4 \mathrm{~Hz}, \mathrm{CH}_{3}\right), 1.23\left(3 \mathrm{H}, \mathrm{d},{ }^{3} J_{H H} 6.4 \mathrm{~Hz}, \mathrm{CH}_{3}\right)$, 1.81-1.95 $\left(4 \mathrm{H}, \mathrm{m}, 2 \mathrm{CH}_{2}\right), 2.16-2.27\left(4 \mathrm{H}, \mathrm{m}, 2 \mathrm{CH}_{2}\right), 2.48-2.61(1 \mathrm{H}, \mathrm{m}, \mathrm{CH}), 3.25\left(1 \mathrm{H}, \mathrm{dd},{ }^{3} \mathrm{~J}_{H H} 2.8,10.4 \mathrm{~Hz}, \mathrm{CH}\right)$, 3.78(1H, d, $\left.{ }^{3} \mathrm{~J}_{H H} 2.8 \mathrm{~Hz}, \mathrm{CH}\right), 7.23-7.27(3 \mathrm{H}, \mathrm{m}, \mathrm{HAr}), 7.36-7.40(2 \mathrm{H}, \mathrm{m}, \mathrm{HAr}) .{ }^{13} \mathrm{C} \mathrm{NMR}(100 \mathrm{MHz}, \mathrm{CDCl} 3): \delta(\mathrm{ppm}):$ 20.4, 21.9, 22.7, 24.2, 30.0, 38.7, 39.0, 39.2, 48.7, 83.7, 87.9, 114.2, 123.1, 128.0, 128.1, 131.8, 163.8, 165.4; HRMS $(m / z):[M+N a]^{+}$calcd for $\mathrm{C}_{20} \mathrm{H}_{22} \mathrm{NaO}_{4}, 349.1416$; found, 349.1408.

5-[1-Phenyl-3-(4-chlorophenyl)-prop-2-yn-1-yl)-2,2-butylidene-1,3-dioxane-4,6-dione (4I). White solid, mp 139-140 ${ }^{\circ} \mathrm{C}$ (Yield: $\left.72 \%\right) .{ }^{1} \mathrm{H}$ NMR $\left(400 \mathrm{MHz}, \mathrm{CDCl}_{3}\right): \delta(\mathrm{ppm}): 1.79-1.92\left(4 \mathrm{H}, \mathrm{m}, 2 \mathrm{CH}_{2}\right), 2.09-2.21\left(4 \mathrm{H}, \mathrm{m}, 2 \mathrm{CH}_{2}\right)$, $4.01\left(1 \mathrm{H}, \mathrm{d},{ }^{3} \mathrm{~J}_{H H} 2.8 \mathrm{~Hz}, \mathrm{CH}_{3}\right), 5.09\left(1 \mathrm{H}, \mathrm{d},{ }^{3} \mathrm{~J}_{H H} 2.8 \mathrm{~Hz}, \mathrm{CH}_{3}\right), 7.26-7.32(3 \mathrm{H}, \mathrm{m}, \mathrm{HAr}), 7.36\left(2 \mathrm{H}, \mathrm{d},{ }^{3} \mathrm{~J}_{H H} 7.6 \mathrm{~Hz}, \mathrm{HAr}\right)$, $7.40\left(2 \mathrm{H}, \mathrm{d},{ }^{3} \mathrm{~J}_{H H} 8.8 \mathrm{~Hz}, \mathrm{HAr}\right), 7.6 .4\left(2 \mathrm{H}, \mathrm{d},{ }^{3} J_{H H} 7.6 \mathrm{~Hz}, \mathrm{HAr}\right) ;{ }^{13} \mathrm{C} \mathrm{NMR}\left(100 \mathrm{MHz}^{\mathrm{C} C D C l}\right): \delta(\mathrm{ppm}): 22.6,24.2$, $35.8,38.5,38.9,53.8,83.9,87.3,114.3,121.3,127.8,128.6,128.8,133.2,134.3,137.0,162.9,164.0$. HRMS calcd for $\mathrm{C}_{23} \mathrm{H}_{19} \mathrm{ClNaO}_{4}[\mathrm{M}+\mathrm{Na}]^{+} 417.0870$, found $\mathrm{m} / \mathrm{z} 417.0862$.

5-[1-n-Propyl-3-(4-Chlorophenyl)-prop-2-yn-1-yl]-2,2-butylidene-1,3-dioxane-4,6-dione (4m). White solid, mp 121-122 ${ }^{\circ} \mathrm{C}$ (Yield: 68\%). ${ }^{1} \mathrm{H}$ NMR $\left(400 \mathrm{MHz}, \mathrm{CDCl}_{3}\right): \delta(\mathrm{ppm}): 0.99\left(3 \mathrm{H}, \mathrm{t},{ }^{3} \mathrm{~J}_{\mathrm{HH}} 7.2 \mathrm{~Hz}, \mathrm{CH}_{3}\right), 1.48-1.61(2 \mathrm{H}, \mathrm{m}$, $\left.\mathrm{CH}_{2}\right), 1.65-1.70\left(1 \mathrm{H}, \mathrm{m}, \mathrm{CH}_{2}\right), 1.84-1.95\left(4 \mathrm{H}, \mathrm{m}, 2 \mathrm{CH}_{2}\right.$, butylidene), 2.09-2.15 $\left(1 \mathrm{H}, \mathrm{m}, \mathrm{CH}_{2}\right), 2.18-2.27(4 \mathrm{H}, \mathrm{m}$, $2 \mathrm{CH}_{2}$, butylidene), 3.60-3.63 $(1 \mathrm{H}, \mathrm{m}, \mathrm{CH}), 3.72\left(1 \mathrm{H}, \mathrm{d},{ }^{3} \mathrm{~J}_{H H} 2.4 \mathrm{~Hz}, \mathrm{CH}\right), 7.24\left(2 \mathrm{H}, \mathrm{d},{ }^{3} J_{H H} 8.0 \mathrm{~Hz}, 2 \mathrm{CH}, \mathrm{HAr}\right), 7.32$ $\left(2 \mathrm{H}, \mathrm{d},{ }^{3} \mathrm{~J}_{\mathrm{HH}} 8.0 \mathrm{~Hz}, 2 \mathrm{CH}, \mathrm{HAr}\right) .{ }^{13} \mathrm{C} \mathrm{NMR}\left(100 \mathrm{MHz}, \mathrm{CDCl}_{3}\right): \delta$ (ppm): 13.6, 21.3, 22.6, 24.3, 30.6, 34.0, 38.4, 39.0, $51.2,82.0,89.3,114.2,121.6,128.5,133.1,134.0,163.8,164.1$. HRMS calcd for $\mathrm{C}_{21} \mathrm{H}_{23} \mathrm{ClNaO}_{4}[\mathrm{M}+\mathrm{Na}]^{+}$ 383.1026; found, 383.1022 .

\section{Acknowledgements}

This work was supported by the Science and Technology research project of Jiangxi Provincial Education Department (No.GJJ170170). We also thank the support from the Graduate Innovation Foundation of Jiangxi Province (No.YC2015-B023).

\section{References}

1. Bharate, S. B.; Nemmani, K. V. S.; Vishwakarma, R. A. Expert. Opin. Ther. Pat. 2009, 19, 237. https://doi.org/10.1517/13543770802665717

2. Nahm, S.; Weinreb, S. M. Tetrahedron Lett. 1981, 22, 3815. https://doi.org/10.1016/S0040-4039(01)91316-4

3. Knopfel, T. F., Carreira, E. M. J. Am. Chem. Soc. 2003, 125, 6054. https://doi.org/10.1021/ja035311z

4. Liu, S.; Fillion, E. Org. Lett. 2014, 16, 5748. https://doi.org/10.1021/ol502811j

5. Jia,W.; Li, S.; Yu, M.; Chen, M.; Jiao, N. Tetrahedron Lett. 2009, 50, 5406. https://doi.org/10.1016/j.tetlet.2009.07.050 
6. Li, S.; Jia, W.; Jiao, N. Adv. Synth. Catal. 2009, 351, 569.

https://doi.org/10.1002/adsc.200800695

7. Imano, T.; Sonia, S.; Esther, D. Arkivoc 2010, (iii), 7.

https://doi.org/10.3998/ark.5550190.0011.302

8. Pappo, R.; Callins, P. W. Tetrahedron Lett. 1972, 13, 2627.

https://doi.org/10.1016/S0040-4039(01)84892-9

9. Sinclair, J. A.; Molander, G. A.; Brown, H. C. J. Am. Chem. Soc. 1977, 99, 954.

https://doi.org/10.1021/ja00445a054

10. Hooz, J.; Layton, R. B. J. Am. Chem. Soc. 1971, 93,7320.

https://doi.org/10.1021/ja00755a037

11. Nagata, W.; Yoshioka, M. Tetrahedron Lett. 1966, 18, 1913.

https://doi.org/10.1016/S0040-4039(00)76271-X

12. Mikael, B.; Magnus, E.; Martin, N.; Thomas, O. J. Org. Chem. 1993, 58, 7238.

https://doi.org/10.1021/jo00077a055

13. Magnus, E.; Tommy, I.; Martin, N.; Thomas, O. J. Org. Chem. 1997, 62, 182.

14. Kim, S., Park, J. H.; Jon, S. Y. Bull. Korean Chem. Soc. 1995, 16, 783.

15. Knopfel, T. F.; Zarotti, P.; Ichikawa, T.; Carreira, E. M. J. Am. Chem. Soc. 2005, 127, 9682.

https://doi.org/10.1021/ja052411r

16. Fujimori, S.; Knopfel, T. F.; Zarotti, P.; Ichikawa, T.; Carreira, E. M.; Boyall, D. Bull. Chem. Soc. Jpn. 2007, 80, 1635.

https://doi.org/10.1246/bcsj.80.1635

17. Fujimori, S.; Carreira, E. M. Angew. Chem. Int. Ed. 2007, 46, 4964.

https://doi.org/10.1002/anie.200701098

18. Zarotti, P.; Knopfel, T. F.; Aschwanden, P.; Carreira, E. M. ACS Catal. 2012, 2, 1232.

https://doi.org/10.1021/cs300146x

19. Mishra, S; Liu, J.; Aponick, A. J. Am. Chem. Soc. 2017, 139, 3352.

https://doi.org/10.1021/jacs.7b00363

20. Knopfel, T. F.; Boyall, D.; Carreira, M. Org. Lett. 2004, 6, 2281.

https://doi.org/10.1021/ol0491585

21. Xu, Z. H.; Chen, F. B.; Li, Y. Y.; Huang, Q. S.; Liao, C. W. Chin. J. Org. Chem. 2018, 38, 3101.

22. Yan, N.; Xiong, B.; Liao, W. L.; Xu, Z. H. Chin. J. Org. Chem. 2010, 30, 1391.

23. Xu, Z. H.; Lin, C. H. Chin. J. Org. Chem. 2013, 33, 1540.

https://doi.org/10.6023/cjoc201211031

24. Xu, Z. H.; Xiong, B.; Lei, Z. W. Chin. J. Jiangxi Normal Uni. (Natl. Sci.) 2013, 37, 337. 